(2) Case report

\section{(ə) OPEN ACCESS}

\title{
Utilisation of advanced MRI techniques to understand neurovascular complications of PHACE syndrome: a case of arterial stenosis and dissection
}

\author{
Laura B Eisenmenger, ${ }^{1}$ Leonardo A Rivera-Rivera, ${ }^{2}$ Kevin M Johnson, ${ }^{1,2}$ Beth A Drolet ${ }^{3}$
}

${ }^{1}$ Radiology, University of

Wisconsin, Madison, Wisconsin, USA

${ }^{2}$ Medical Physics, University of Wisconsin, Madison, Wisconsin, USA

${ }^{3}$ Dermatology, University of Wisconsin, Madison, Wisconsin, USA

\section{Correspondence to} Dr Laura B Eisenmenger; leisenmenger@uwhealth.org

Accepted 31 August 2020
Check for updates

(c) BMJ Publishing Group Limited 2020. Re-use permitted under CC BY-NC. No commercial re-use. See rights and permissions. Published by BMJ.

To cite: Eisenmenger $L B$, Rivera-Rivera LA, Johnson KM, et al. BMJ Case Rep 2020;13:e235992. doi:10.1136/bcr-2020235992

\section{SUMMARY}

PHACE syndrome is a rare disorder with posterior fossa brain malformations, segmental infantile haemangiomas, arterial anomalies, cardiac defects and eye anomalies. Cerebral and cervical arterial abnormalities occur commonly in these patients, predisposing subjects with PHACE syndrome to neurovascular complications including migraine-like headaches, moyamoya vasculopathy, arterial dissection and arterial ischaemia stroke. We leveraged institutional MRI protocols developed for adult neurovascular disease to better elucidate the pathogenesis of the arterial alternations observed in PHACE. Using high-resolution vessel wall and 4D flow MRI, we demonstrated enhancement, focal dissection and altered blood flow in a 7-year-old girl with PHACE syndrome. This is the first-time vessel wall imaging has been used to detail the known arterial changes in PHACE, and these findings may indicate that progressive vascular narrowing and vessel wall changes/ inflammation are a factor in chronic headaches and other arterial complications seen in subjects with PHACE syndrome.

\section{BACKGROUND}

PHACE syndrome is an acronym to describe a rare neurovascular disorder with posterior fossa brain malformations, segmental infantile haemangiomas, arterial anomalies, cardiac defects and eye anomalies. ${ }^{1}$ Cerebral and cervical arterial abnormalities are the most common non-cutaneous anomaly in PHACE syndrome occurring in over $85 \%$ of patients, including vessel narrowing, dysgenesis, anomalous course and/or origin, and non-visualisation. ${ }^{2}$ These neurovascular anomalies predispose infants and children to highly morbid complications including headaches, ${ }^{3}$ moyamoya vasculopathy (MMV), ${ }^{4}$ arterial dissection, ${ }^{5} 6$ transient ischaemic attacks (TIAs) and arterial ischaemia stroke (AIS). ${ }^{7}$ While there is anecdotal evidence that progressive vascular narrowing and vessel wall abnormalities may be a factor in these neurovascular complications, no study or case report to date has characterised the vessel wall in PHACE syndrome, specifically not in a case of PHACE syndrome with documented, progressive vascular stenosis. In addition, there is no evidence in the literature for vascular inflammation playing a potential role in the progressive arterial abnormalities that can occur in these subjects. We present a case of PHACE syndrome in a child with chronic migraine-like headaches and known progressive vascular narrowing using both highresolution vessel wall and 4D flow MRI.

\section{CASE PRESENTATION}

The patient is a 7-year-old girl with previously diagnosed PHACE syndrome presenting for both clinical evaluation and surveillance imaging. She originally presented at birth with a sternal cleft and an infantile haemangioma on the left aspect of the upper and lower lips as well as involving the adjacent oral mucosa. The patient has had surgical resection of residual fibrofatty tissue on the lower lip and pulsed dye laser therapy to the lips, cheek and nose. A screening echocardiogram documented mild aortic arch hypoplasia without blood flow limitation. Prior cervical and intracranial vascular imaging has demonstrated left internal carotid artery (ICA) narrowing involving the distal aspect of the left cervical ICA extending into the carotid canal. Collateral blood supply to the left anterior circulation is supplied by a prominent left posterior communicating artery (PCOMM).

The patient was interviewed with her mother, both reporting she has three to four migraine-like headaches per week which are often occupied by nausea and blurry vision. These headaches limit her activities during their occurrence with an average of 1-2 missed school days per week. Given her preexisting vascular disease, no vasoactive medications have been initiated, and the family usually treats the headaches with rest, hydration and over-thecounter pain medications. No prescription medications have been used.

\section{INVESTIGATIONS}

MRI with angiography (MRA) was performed in December 2019 with inclusion of 4D flow MRI and high-resolution vessel wall imaging. 4D flow MRI is a technique which encodes the velocity field in the image phase, providing both volumetric angiography and quantitative assessment of blood flow velocities throughout the cardiac cycle. ${ }^{8}$ While scan times for 4D flow MRI have been prohibitive in the past, recent advances in accelerated imaging have made these techniques available for comprehensive neurovascular haemodynamic assessment. Studies have demonstrated the capability to derive advanced haemodynamic parameters from the velocity field captured using 4D flow, including measures of wall 


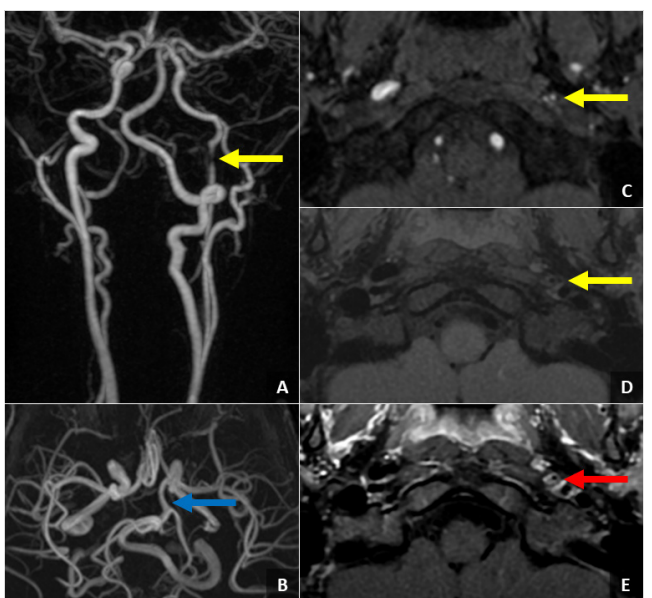

Figure 1 Time-resolved imaging of contrast MRI with angiography (MRA). (A) Time-of-flight MRA maximum intensity projection, $(B, C)$ pre-contrast T1-CUBE, a 3-dimensional (3D) fast spin-echo (FSE)-based, black blood MRI sequence, and (D) post-contrast T1-CUBE images (E) demonstrating severe narrowing of the left cervical internal carotid artery near the skull base (yellow arrows) with associated concentric vessel wall enhancement (red arrow) in a 7-year-old girl with PHACE syndrome complicated by headaches three to four times per week. Collateral blood flow to the left internal carotid artery distribution is supplied by a large left posterior communicating artery (blue arrow).

shear stress, pulse wave velocity (PWV), pressure gradients and vessel tracking. ${ }^{9}$

On structural imaging, fullness and heterogeneous signal was present involving the left aspect of the upper and lower lips, consistent with the reported, treated haemangioma at this location. The brain parenchyma was normal with normal appearance of the posterior fossa.

On 3D time-of-flight and post-contrast time-resolved imaging of contrast kinetics MRA, there was redemonstration of luminal narrowing of the left distal cervical ICA extending into the carotid canal (figure 1). At the site of greatest narrowing, the ICA measures $1.5 \mathrm{~mm}$ in diameter, representing approximately $60 \%$ stenosis, mildly progressed compared with the most recent prior in April 2015 at which time the stenosis was approximately $50 \%$. There is reconstitution of left ICA calibre in the petrous segment with additional collateral filling of the anterior circulation via a prominent left PCOMM artery. Otherwise, there is notable diffuse dolichoectasia of the intracranial vasculature without additional focal cervical or intracranial arterial or venous abnormalities.

On post-contrast, high-resolution vessel wall imaging (figure 1), there is wall thickening of the left distal cervical ICA segment in the areas of luminal narrowing with associated vessel wall enhancement. Intracranially, there is a focal, linear filling defect in the left petrous ICA, concerning for a small, focal dissection with mild focal stenosis (figure 2). No other areas of vessel wall abnormality are present without any additional vessel wall thickening or enhancement.

On 4D flow MRI acquired using the previously described accelerated 3D radial technique, ${ }^{8} 9$ velocity images (figure 3 ) displayed reduced velocities in the left cervical ICA compared with right cervical ICA and basilar artery. Further, higher velocities were observed in the prominent left PCOMM artery. Blood flow rates quantified from velocity data were: $1.31 \pm 0.64 \mathrm{~mL} / \mathrm{s}$ in the left cervical ICA, $4.26 \pm 1.76 \mathrm{~mL} / \mathrm{s}$ in the right cervical ICA, $4.44 \pm 1.58 \mathrm{~mL} / \mathrm{s}$ in the basilar artery and $2.16 \pm 0.62$

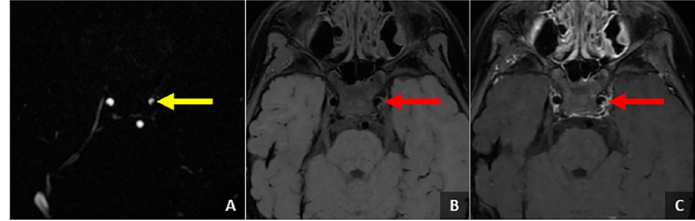

Figure 2 Time-of-flight MRI with angiography maximum intensity projection. (A) Pre-contrast T1-CUBE and (B) post-contrast T1-CUBE images (C) show a mild, focal narrowing (yellow arrow) with a linear filling defect in the left petrous internal carotid artery concerning for a small, focal dissection (red arrows), which can be seen in the setting of PHACE syndrome.

$\mathrm{mL} / \mathrm{s}$ in the left PCOMM artery. From the cardiac-resolved flow waveforms, pulsatility indices (PIs) were estimated $(\mathrm{PI}=(\mathrm{Qmax}-\mathrm{Qmean}) / \mathrm{Qmean}, \mathrm{Q}=$ flow $))$. PI was largest in the left cervical ICA $(P I=1.48 \pm 0.16)$, followed by right cervical ICA $(\mathrm{PI}=1.38 \pm 0.05)$. The PI in the basilar artery was $1.03 \pm 0.09$ and $1.02 \pm 0.05$ in the left PCOMM artery. By estimating the temporal shift of the cardiac waveform along the arteries, PWV was quantified as previously described ${ }^{10}$ using the relationship $\mathrm{PWV}=\Delta \mathrm{x} / \Delta \mathrm{t}$, where $\Delta \mathrm{x}$ is the distance travelled by the pulse wave and $\Delta \mathrm{t}$ is the time of traversal. The PWV in the left cervical ICA was $2.9 \mathrm{~m} / \mathrm{s}$ and $7.3 \mathrm{~m} / \mathrm{s}$ in the right cervical ICA.

\section{TREATMENT}

Consensus guidelines for the prevention of neurovascular complications were published in 2016. ${ }^{11}$ Daily aspirin was

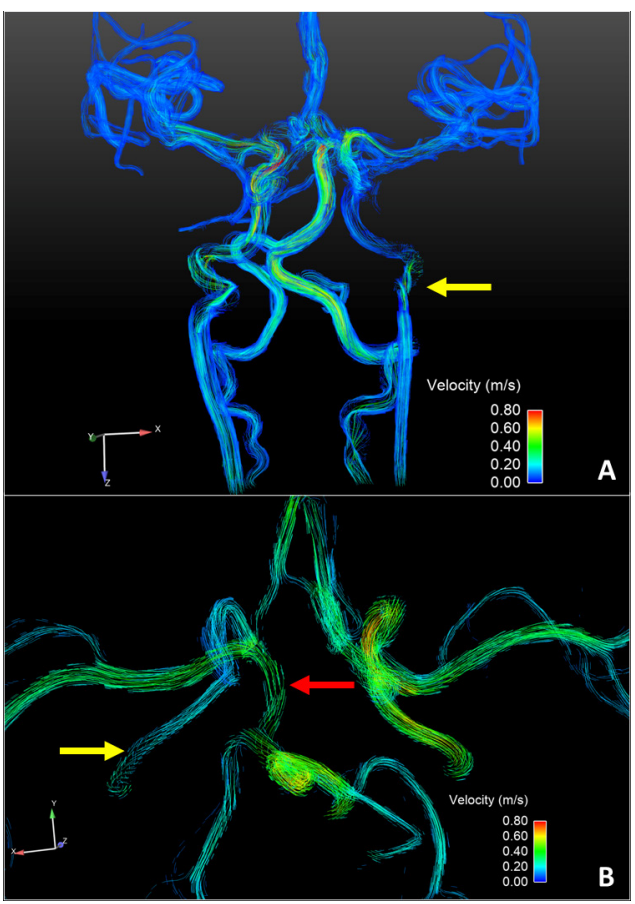

Figure 3 Coronal (A) and axial (B) views of blood flow and distribution on the Circle of Willis derived from 4D flow data. Decreased velocities were measured in the left cervical internal carotid artery (ICA) on the side of stenosis (yellow arrows) while increased velocities were measured in the prominent left posterior communicating (PCOMM) artery (red arrow). The left PCOMM is providing the majority of the blood supply to the left anterior cerebral and middle cerebral artery distributions, compensating for the decreased blood flow through the left ICA. 
recommended for patients at high risk for acute ischaemic stroke. High risk was defined as: (1) significant narrowing $(>25 \%)$ or occlusion of principal cerebral vessels within or above the Circle of Willis that results in an 'isolated' circulation, (2) tandem or multiple arterial stenoses associated with complex blood flow that may potentially result in diminished cerebral perfusion, (3) imaging findings in the brain parenchyma suggestive of chronic or silent ischaemia, or progressive stenoocclusive disease. ${ }^{11}$ Imaging findings from conventional MRI/ MRA imaging performed on our patient did not meet criteria for aspirin therapy; however, after identification of the vessel wall enhancement and dissection she was started on $81 \mathrm{mg}$ of aspirin each day.

\section{OUTCOME AND FOLLOW-UP}

Within 4 weeks of starting aspirin, the patient's headaches improved. She experienced a decrease in both frequency and severity of her headaches. The improved headache symptoms have persisted through to the most recent 3-month follow-up. No complications including TIA or stroke have resulted from the left ICA stenosis or focal dissection to date.

\section{DISCUSSION}

With the utilisation of advanced high-resolution vessel wall imaging and 4D flow MRA, we are able to detect vessel wall enhancement and altered blood flow in an arterial segment with known progressive luminal narrowing as well as detect a focal dissection not visible on more conventional vascular imaging sequences. This is the first time these advanced imaging techniques have been used in a patient with PHACE syndrome and progressive arteriopathy. These imaging findings directly led to the addition of aspirin therapy despite not meeting the criteria for aspirin per the consensus guidelines for the prevention of neurovascular complications, ${ }^{11}$ with notable improvement in the subject's headaches after beginning treatment.

PHACE is an acronym coined by Frieden et al for posterior fossa, haemangiomas, arterial, cardiovascular, eye anomalies and ventral developmental defects. ${ }^{1}$ Though the acronym PHACE is also used sometimes due to the presence of sternal abnormalities, such as sternal cleft present in this case. While the underlying aetiology of PHACE syndrome remains unknown, many believe that the clinical manifestations of the syndrome are downstream events that originate from developmental defects of small-sized and medium-sized arteries of the head and neck. Given the ipsilateral and segmental nature of the clinical features, post-zygotic somatic mutation(s) have been proposed; however, extensive genomic investigation has not yet identified pathogenic mutations shared by patients.

Although the most apparent and often presenting feature of PHACE is infantile haemangioma, the long-term morbidity is the result of the arterial and aortic arch abnormalities observed in this syndrome. Arterial anomalies are extraordinarily common in PHACE and approach or exceed the incidences observed in wellknown connective tissue disorders such as Marfan's syndrome. ${ }^{2}$ Over $85 \%$ of patients with PHACE are born with abnormalities of cervical and cerebral arteries, and progressive changes may lead to devastating neurovascular complications. ${ }^{2}$ As existing PHACE cohorts reach adolescence and adulthood, these complications of PHACE are increasingly being recognised and include migraine-like headaches, ${ }^{3}$ TIAs, AIS, ${ }^{7} \mathrm{MMV}^{4}$ and rarely arterial dissection. ${ }^{5}$ Although we know patients are at increased risk of these emerging neurovascular-related morbidities, very little is
Patient's perspective

Once we saw the imaging, it helped give us a really good idea of what is going on and may explain why she has been experiencing headaches so frequently lately. My daughter is at the age where she is more aware of the changes and the differences that are going on inside of her. Once we found out about the dissection, she was sad because she knew that she would still be restricted from doing the activities she loves and excels at doing like gymnastics, but she also could comprehend why she cannot perform those activities. She was also scared because she can't fully understand what is going on. The doctors did a great job talking to her, and now she knows the importance of taking care of herself and complying with taking aspirin on a daily basis. Seeing the 4D flow really helped put everything into perspective. It's incredible to see how everything is working. We were able to see the jagged structure of the internal carotid artery and the size and flow of the posterior communicating artery. My daughter still feels sad that the problem exists, but she is able to talk to her friends and family about what is going on. Knowing the dissection exists is incredibly nerve racking and something that is constantly on our minds, but we know it is there and we are able to make people she is around on a regular basis know that if she complains of any symptoms that aren't normal to contact emergency officials right away. This protocol could detect findings in other patients with PHACE and is so valuable. We are now feeling strength and hope in knowing where the abnormalities exist and feel confident that we are doing all we can.

\section{Learning points}

- Vessel wall enhancement may be seen in areas of progressive vascular narrowing, potentially indicating an area of vascular remodelling and/or active inflammation.

- High-resolution vessel wall imaging could assist in early detection of arterial dissection.

- Blood flow alterations may be seen in areas of vascular abnormalities; however, additional studies with 4D flow are needed to characterise blood flow in normal vessel segments, congenitally abnormal vessels and vessel segments with progressive changes to fully understand the implications of these findings.

- Progressive vascular narrowing and vessel wall changes/ inflammation could be a factor in chronic headaches and other arterial complications seen in subjects with PHACE syndrome.

known about the nature of the arteriopathy (ie, no good imaging and no pathology).

Despite this being the first reported case of vessel wall enhancement in PHACE syndrome, other similar arterial pathologies have documented vessel wall enhancement, supporting our findings. For example, enhancement has been seen in cases of other causes of secondary MMV, ${ }^{12}$ idiopathic moyamoya disease, ${ }^{13}{ }^{14}$ both paediatric ${ }^{15}$ and adult AIS, ${ }^{16}{ }^{17}$ and multiple types of vasculitides. ${ }^{18} 19$ Specifically, in the paediatric population, the Vascular Effects of Infection in Pediatric Stroke Study was the largest study evaluating stroke in the paediatric population, prospectively enrolling 355 children with arterial ischaemic stroke (2010-2014). ${ }^{15}$ This study not only documented vessel 
wall enhancement in paediatric cases of local vascular inflammation, but also recognised the potential importance of using advanced vascular techniques in investigation of other childhood arteriopathy subtypes.

By measuring the flow velocity field, 4D flow MRI allows the characterisation of haemodynamic parameters including average blood flow rates, PIs, PWV, pressure gradients, wall shear stress, vessel tracking and other parameters. ${ }^{9}$ 4D flow MRI has been used to detect alterations in intracranial blood flow in multiple types as vascular lesions including aneurysms, ${ }^{20} 21$ dural arteriovenous fistulas, ${ }^{22}$ vein of Galen malformations ${ }^{23}$ and arteriovenous malformations ${ }^{24}$ as well as more diffuse neurological disease such as Alzheimer's disease ${ }^{102526}$; however, to the best of our knowledge, this is the first case of PHACE syndrome evaluated with this advanced technique. As expected, we found decreased blood flow through the stenotic left ICA and increased blood flow through the prominent left PCOMM artery. We also found higher PWVs in the right ICA without narrowing compared with the narrowed left ICA. This could be due to a combination of effects including compensatory mechanisms driving increased blood flow velocities through the right ICA, and vascular resistance along the left ICA; however, larger studies using 4D flow MRI in PHACE are needed to further investigate these findings.

Although this is only one PHACE syndrome case of vessel wall enhancement, focal dissection and altered blood flow in an arterial segment with known progressive narrowing, these findings could suggest a degree of active vessel wall remodelling and possibly even vessel wall inflammation. The subject in this case report has severe headaches impacting her school performance and personal life which improved with the addition of aspirin therapy. There is a possibility that vascular narrowing, remodelling, and/or inflammation could be playing a role in her headaches as well as their severity. While one case does not prove that vessel wall enhancement and blood flow alterations will be present in all subjects with PHACE with progressive vessel wall narrowing, further studies are warranted to investigate this relationship as well as the relationship between vessel wall enhancement and blood flow to chronic headaches, MMV, arterial dissection and AIS seen in populations with PHACE syndrome.

\section{Acknowledgements The project described was supported by the Clinical and Translational Science Award (CTSA) program, through the NIH National Center for Advancing Translational Sciences (NCATS), grant UL1TR002373 and KL2TR002374. \\ Contributors LBE: clinical neuroradiologist and vascular researcher primarily responsible for imaging evaluation and patient diagnosis, evaluation of image quality, principle writer of the manuscript, and manuscript editing. LAR: medical physicist primarily responsible for analysis of $4 \mathrm{D}$ flow images, as well as manuscript review and editing. KMJ: medical physicist primarily responsible for development and optimisation of the imaging sequences used in this study, as well as manuscript review and editing. BAD: clinical dermatologist primarily treating the patient, responsible for physical and clinical examinations as well as treatment and follow-up, and manuscript review and editing.}

Funding The authors have not declared a specific grant for this research from any funding agency in the public, commercial or not-for-profit sectors.

Disclaimer The content is solely the responsibility of the authors and does not necessarily represent the official views of the $\mathrm{NIH}$.

Competing interests None declared.

Patient consent for publication Parental/guardian consent obtained.

Provenance and peer review Not commissioned; externally peer reviewed.

Open access This is an open access article distributed in accordance with the Creative Commons Attribution Non Commercial (CC BY-NC 4.0) license, which permits others to distribute, remix, adapt, build upon this work non-commercially, and license their derivative works on different terms, provided the original work is properly cited and the use is non-commercial. See: http://creativecommons.org/ licenses/by-nc/4.0/.

\section{REFERENCES}

1 Frieden IJ, Reese V, Cohen D. PHACE syndrome. the association of posterior fossa brain malformations, hemangiomas, arterial anomalies, coarctation of the aorta and cardiac defects, and eye abnormalities. Arch Dermatol 1996;132:307-11.

2 Hess CP, Fullerton HJ, Metry DW, et al. Cervical and intracranial arterial anomalies in 70 patients with PHACE syndrome. AJNR Am J Neuroradiol 2010;31:1980-6.

3 Yu J, Siegel DH, Drolet BA, et al. Prevalence and clinical characteristics of headaches in PHACE syndrome. J Child Neurol 2016;31:468-73.

4 Schilter KF, Steiner JE, Demos W, et al. RNF213 variants in a child with PHACE syndrome and moyamoya vasculopathy. Am J Med Genet A 2017;173:2557-61.

5 Stefanko NS, Cossio M-L, Powell J, et al. Natural history of PHACE syndrome: a survey of adults with PHACE. Pediatr Dermatol 2019;36:618-22.

6 Kulyk C, Farina F, Palmieri A, et al. "Phacing" a New Cause of Carotid Artery Dissection. Neurologist 2017;22:54-6.

7 Siegel DH, Tefft KA, Kelly T, et al. Stroke in children with posterior fossa brain malformations, hemangiomas, arterial anomalies, coarctation of the aorta and cardiac defects, and eye abnormalities (PHACE) syndrome: a systematic review of the literature. Stroke 2012;43:1672-4.

8 Johnson KM, Lum DP, Turski PA, et al. Improved 3D phase contrast MRI with offresonance corrected dual echo VIPR. Magn Reson Med 2008;60:1329-36.

9 Turski PA, Levine R, Turnipseed W, et al. MR angiography flow analysis. neurovascular applications. Magn Reson Imaging Clin N Am 1995;3:541-55.

10 Rivera-Rivera LA, Cody KA, Eisenmenger $L$, et al. Assessment of vascular stiffness in the internal carotid artery proximal to the carotid canal in Alzheimer's disease using pulse wave velocity from low RANK reconstructed 4D flow MRI. J Cereb Blood Flow Metab 2020:271678X20910302.

11 Garzon MC, Epstein LG, Heyer GL, et al. PHACE syndrome: Consensus-Derived diagnosis and care recommendations. J Pediatr 2016;178:24-33.

12 Mossa-Basha M, de Havenon A, Becker KJ, et al. Added value of vessel wall magnetic resonance imaging in the differentiation of moyamoya vasculopathies in a non-Asian cohort. Stroke 2016;47:1782-8.

13 Kathuveetil A, Sylaja PN, Senthilvelan S, et al. Vessel wall thickening and enhancement in high-resolution intracranial vessel wall imaging: a predictor of future ischemic events in moyamoya disease. AJNR Am J Neuroradiol 2020;41:100-5.

14 Chen X, Wang J, Liu Y, et al. Proximal internal carotid artery stenosis associates with diffuse wall thickening in petrous arterial segment of moyamoya disease patients: a three-dimensional magnetic resonance vessel wall imaging study. Neuroradiology 2019;61:29-36.

15 Wintermark M, Hills NK, DeVeber GA, et al. Clinical and imaging characteristics of arteriopathy subtypes in children with arterial ischemic stroke: results of the VIPS study. AJNR Am J Neuroradiol 2017:38:2172-9.

16 Dlamini N, Yau I, Muthusami P, et al. Arterial wall imaging in pediatric stroke. Stroke 2018:49:891-8

17 de Havenon A, Mossa-Basha M, Shah L, et al. High-Resolution vessel wall MRI for the evaluation of intracranial atherosclerotic disease. Neuroradiology 2017:59:1193-202.

18 Mossa-Basha M, Shibata DK, Hallam DK, et al. Added value of vessel wall magnetic resonance imaging for differentiation of Nonocclusive intracranial vasculopathies. Stroke 2017;48:3026-33.

19 Mossa-Basha M, Hwang WD, De Havenon A, et al. Multicontrast high-resolution vessel wall magnetic resonance imaging and its value in differentiating intracranial vasculopathic processes. Stroke 2015;46:1567-73.

20 Moftakhar R, Aagaard-Kienitz B, Johnson K, et al. Noninvasive measurement of intraaneurysmal pressure and flow pattern using phase contrast with vastly undersampled isotropic projection imaging. AJNR Am J Neuroradio/ 2007;28:1710-4.

21 Liu J, Koskas L, Faraji F, et al. Highly accelerated intracranial 4D flow MRI: evaluation of healthy volunteers and patients with intracranial aneurysms. MAGMA 2018;31:295-307

22 Rivera-Rivera LA, Johnson KM, Turski PA, et al. Pressure mapping and hemodynamic assessment of intracranial dural sinuses and dural arteriovenous fistulas with 4D flow MRI. AJNR Am J Neuroradiol 2018;39:485-7.

23 Li Y, Ahmed R, Rivera-Rivera LA, et al. Serial quantitative and qualitative measurements of flow in vein of Galen malformations using 4-Dimensional flow magnetic resonance imaging (phase contrast Vastly undersampled isotropic projection). World Neurosurg 2019;126:405-12.

24 Chang W, Wu Y, Johnson K, et al. Fast contrast-enhanced 4D MRA and 4D flow MRI using constrained reconstruction (HYPRFlow): potential applications for brain arteriovenous malformations. AJNR Am J Neuroradio/ 2015;36:1049-55.

25 Berman SE, Clark LR, Rivera-Rivera LA, et al. Intracranial arterial 4D flow in individuals with mild cognitive impairment is associated with cognitive performance and amyloid positivity. J Alzheimers Dis 2017:60:243-52.

26 Rivera-Rivera LA, Schubert T, Turski P, et al. Changes in intracranial venous blood flow and Pulsatility in Alzheimer's disease: a 4D flow MRI study. J Cereb Blood Flow Metab 2017:37:2149-58. 
Copyright 2020 BMJ Publishing Group. All rights reserved. For permission to reuse any of this content visit https://www.bmj.com/company/products-services/rights-and-licensing/permissions/

BMJ Case Report Fellows may re-use this article for personal use and teaching without any further permission.

Become a Fellow of BMJ Case Reports today and you can:

- Submit as many cases as you like

- Enjoy fast sympathetic peer review and rapid publication of accepted articles

- Access all the published articles

Re-use any of the published material for personal use and teaching without further permission

Customer Service

If you have any further queries about your subscription, please contact our customer services team on +44 (0) 2071111105 or via email at support@bmj.com.

Visit casereports.bmj.com for more articles like this and to become a Fellow 